Sessions d'étude - Société canadienne d'histoire de l'Église catholique

\title{
Le diocèse de Sault Ste-Marie depuis 1958
}

\section{Alexandre Carter}

Volume 42, 1975

URI : https://id.erudit.org/iderudit/1007235ar

DOI : https://doi.org/10.7202/1007235ar

Aller au sommaire du numéro

Éditeur(s)

Les Éditions Historia Ecclesiæ Catholicæ Canadensis Inc.

ISSN

0318-6172 (imprimé)

1927-7067 (numérique)

Découvrir la revue

Citer cet article

Carter, A. (1975). Le diocèse de Sault Ste-Marie depuis 1958. Sessions d'étude -

Société canadienne d'histoire de l'Église catholique, 42, 28-42.

https://doi.org/10.7202/1007235ar

Tous droits réservés ㄷ Les Éditions Historia Ecclesiæ Catholicæ Canadensis Inc., 1976
Ce document est protégé par la loi sur le droit d'auteur. L’utilisation des services d'Érudit (y compris la reproduction) est assujettie à sa politique d'utilisation que vous pouvez consulter en ligne.

https://apropos.erudit.org/fr/usagers/politique-dutilisation/ 


\section{Le diocèse de Sault Ste-Marie depuis 1958}

Faire le bilan d'un diocèse aussi complexe que le nôtre n'est pas une tâche facile. La tâche me paraît même plus difficile quand je me rends compte que je m'adresse aux membres d'une société historique, donc à des personnes habituées aux faits et non pas aux simples impressions plus ou moins exactes qu'on peut retenir des années passées. Certaines question se posent immédiatement. Parlet-on de l'Église locale dans sa forme purement institutionnelle? S'agit-il de fournir certaines données comme par exemple le nombres d'églises, de presbytères ou encore de centres quelconques bâtis pendant les dix-huit années de mon ministère? Un tel développement ne me sourit aucunement. Il fausserait en somme la nature même d'un diocèse, d'une Église locale. Faudrait-il alors parler uniquement de l'Église charismatique, de l'Église communauté croyante, priante, Église d'amour et de service? Mais comment examiner adéquatement la vie spirituelle du peuple de Dieu, vie pleine de richesses spirituelles individuelles et communes? Dieu seul serait en mesure de nous révéler une telle histoire.

Il faut donc dès le début poser certaines limites et accepter certaines hypothèses pour bien comprendre ce à quoi je m'engage.

1 - Je parlerai de notre Église toute entière - telle qu'elle existe dans ce monde. Église-institution; Église-charismatique; peuple de Dieu.

2-Il m'est impossible de faire ce bilan d'une façon complètement objective et détachée. Je parle de l'Église dont je suis le pasteur - de l'Église que j'aime. Je la vois avec les yeux d'un évêque. S'attendre à ce que je sois complètement objectif, c'est comme demander à un père de famille de rester purement objectif au sujet de sa propre famille. Donc les paroles que je vous adresse révéleront certains préjugés de ma part. Je laisse à l'histoire de porter les correctifs qui s'imposeront dans un avenir lointain ou rapproché. La vraie histoire exige le passage des années. Sans prétentions je vous expose sincèrement, simplement et aussi concrètement que possible ce qui me paraît les grandes lignes du développement 
du diocèse du Sault Ste-Marie pendant les années durant lesquelles j'ai eu le privilège et l'honneur d'être son pasteur.

3 -Enfin, puisque je m'adresse à la section française de la Société Canadienne d'Histoire de l'Église Catholique, je porterai un intérêt particulier au secteur francophone tout en l'insérant dans l'optique de toute l'histoire de l'Église locale durant les années 1958 à 1975.

Notre Église comme toutes les Églises du monde et comme l'Église catholique universelle dont elle fait partie a vécu des années mouvementées et a connu des expériences inattendues et, pour beaucoup, renversantes. Pour mieux analyser ces années, voici le plan que j'ai l'intention de suivre.

1 - J'essaierai de vous tracer les grandes lignes de notre histoire telle que je la vois pendant trois périodes.

a) L'Église avant le Concile œcuménique Vatican II;

b) Notre Église pendant le Concile;

c) Notre Église pendant les années post-conciliaires.

Je fais cette distinction non seulement pour fin de clarté et de facilité mais parce que je crois que les événements ont grandement influencé la vie et le cheminement de notre Église pendant ces époques. Il est entendu qu'il n'y a pas de cloison étanche entre ces trois périodes. II faut admettre une certaine continuité. Il reste cependant que l'avènement du Concile nous a obligés de repenser notre pastorale et d'affronter de nouveaux problèmes qu'on avait pressentis peut-être mais qui se posaient alors dans toute leur force et avec une insistance irrécusable.

2-En terminant mon exposé j’oserai donner un bref aperçu général $\mathrm{du}$ diocèse prenant en considération ses points faibles et ses points forts, ce qu'on peut espérer, ce qu'on doit craindre.

\section{Notre Eglise avant le Concile}

En 1957, le diocèse du Sault Ste-Marie se trouvait en plein essor. La construction d'après-guerre venait de créer une demande mondiale pour les minéraux qui font la richesse de notre territoire. Une nouvelle ville se bâtissait à Elliot Lake après la découverte de grands gisements d'uranium. La compagnie INCO embauchait à 
Sudbury des centaines de mineurs, et la compagnie Algoma Steel projetait de nouvelles additions à son usine au Sault. L'Église locale sentit l'effet de cette croissance. Après cinquante-trois ans, le diocèse était devenu plus qu'une suite de villages le long du chemin de fer; Sudbury, Sault Ste-Marie et North Bay formaient une chaîne de grands centres desservis par des paroisses dont plusieurs étaient florissantes.

Sous le premier évêque, Monseigneur Scollard, l'histoire en fut une de consolidation. Monseigneur Scollard fut vraiment un évêque missionnaire. Le territoire immense du diocèse, des moyens de communication assez rudimentaires imposèrent d'immenses sacrifices. Par la suite Monseigneur Dignan, successeur de Monseigneur Scollard, a connu lui aussi au début les mêmes difficultés. La dépression économique des années ' 30 se trouvait alors à son apogée. Le diocèse était pauvre, il a fallu surveiller de près les finances et restreindre les dépenses autant que possible. Son fardeau cependant s'allégea dans les années '50, d'abord par la création du diocèse de Fort William (maintenant Thunder Bay) ce qui a réduit le diocèse du Sault à des dimensions plus humaines, et ensuite par l'essor économique qui suivit la guerre. Malheureusement une maladie sérieuse l'a empêché de bénéficier de ces améliorations ou de profiter des nouvelles opportunités qui se présentaient. Ces deux hommes avec leurs qualités et leurs faiblesses se sont sûrement sacrifiés pour l'Église. Ce qui explique un peu le sens profond de la foi qui existait parmi le clergé et le peuple du diocèse du Sault Ste-Marie en 1958.

En arrivant j'ai trouvé un clergé dévoué et courageux. Pauvres pour la plupart, ils ne cédèrent devant aucune difficulté. Plusieurs d'entre eux desservaient des missions sur une étendue de cinquante et cent milles et, faute de routes, ils étaient forcé d'utiliser des moyens de transport assez épuisants. Dévoués et loyaux, ils avaient établi des liens étroits avec leurs paroissiens. Et que dire des communautés religieuses d'hommes et de femmes qui s'étaient dévouées à bâtir et à entretenir des écoles et des institutions, et cela la plupart du temps à même leurs propres ressources. Avec des leaders de ce calibre il n'est pas étonnant de trouver des fidèles attachés à l'Église. Leur foi était simple mais profonde.

Vous allez dire, mais c'est le Paradis que vous décrivez. Pas tout à fait. À part les faiblesses d'individus qu'on trouve partout 
dans n'importe quelle institution humaine un grand malaise existait dans le diocèse. La population francophone ne se sentait pas à l'aise dans la situation qu'on lui avait faite. Je n'ai ni le droit ni l'intention de porter un jugement sur qui que ce soit. Le fait est, un malaise existait. Je l'ai constaté immédiatement. $\mathrm{Si}$ dans la suite je me suis aperçu que quelques-uns exagéraient la situation, je me suis rendu compte que la population francophone souffrait de certaines injustices flagrantes. Aussi la maladie de Monseigneur Dignan avait empêché la mise en œuvre des initiatives qui s'avéraient non seulement nécessaires mais urgentes pour le bien du diocèse. En novembre 1957, le Saint Siège me nomma Administrateur. On entama des discussions à travers le diocèse et on choisit certaines priorités. Tous se mettaient au travail.

1 - Il fallait réconcilier l'élément français et l'élément anglais dans le diocèse. Pour cela on devait énoncer quelques principes, créer certaines structures qui rassureraient la population francophone et lui fourniraient le moyen de s'épanouir et de jouir d'une autonomie juste et raisonnable. De bonnes paroles ne suffisaient plus, il fallait passer à l'action.

2-Tous étaient d'accord pour réorganiser le diocèse de fond en comble. Les comités ou les commissions tel qu'inscrits dans le Canada Ecclésiastique n'existaient que de nom. Il s'agissait donc de créer de nouvelles structures, de nommer des responsables et d'initier une pastorale qui tiendrait compte de la nature et du caractère de notre diocèse.

3 - La simple justice exigera une réforme substantielle du financement du diocèse. Le salaire des prêtres, la contribution des paroisses à l'administration diocésaine n'accusaient aucun principe de base. Des arrangements privés prêtaient à des inégalités pour ne pas dire à des injustices.

4-Il fallait créer un sens social aigu auprès de notre population catholique. La vie paroissiale s'avéra assez florissante quant à la piété personnelle mais le sens du bien commun du diocèse et de la responsabilité envers tous les pauvres semblait manquer. D'où le besoin d'une éducation solide.

Vous pouvez vous imaginer que tout cela était de nature à engager les efforts de tous les fidèles de bonne volonté. 


\section{1 - D'abord la réconciliation :}

a) au niveau des principes je ne fais que citer une lettre pastorale que j'ai écrite le 25 janvier 1958 (Circ. 5, p. 2, par. 2 ...) "Notre diocèse aussi a un arrière-plan et un but déterminé. Pour qu'il n'y ait aucun doute parmi les fidèles ou dans tous les milieux, résumons la situation une fois de plus. Le diocèse du Sault Ste-Marie est un diocèse bilingue. Deux grandes cultures sont à la portée de nos gens s'ils se montrent assez sages pour en prendre avantage. Quoiqu'il en soit, nous désirons énoncer que nous considérons tous nos diocésains comme nos propres enfants, et tous ont droit à la même affection et à la même attention. Les nouveaux Canadiens choisiront naturellement l'une des deux langues officielles de notre pays ou peut-être même les deux; ils sont aussi fortement encouragés à conserver leur langue maternelle et leur culture. Des écoles bilingues et des paroisses françaises existent dans notre diocèse non par tolérance ou privilège, mais par justice. Nous protégerons ce droit comme un devoir de justice. Nous déclarons ces choses clairement pour la première et dernière fois, conscient que nous ne faisons que remplir notre obligation en tant qu'Évêque catholique et que, en agissant ainsi, nous nous conformons aux désirs du Saint-Siège ...»

\section{2 - Réorganisation :}

Ensemble nous avons décidé de limiter la réorganisation du diocèse aux vrais besoins de l'Église locale. On voulait éviter à tout prix de créer une bureaucratie, l'ennemi fatal de la vraie vie de l'Église. Il fallait aussi respecter la réalité des régions géographiques. Des directeurs diocésains et comités, il en fallait. Mais nous avons essayé surtout d'engager des prêtres qui avaient des aptitudes et des expériences dans les domaines divers.

Nous devions tenir compte également du caractère biculturel de notre Église. Après les premiers chocs des déclarations de principe, les plus ouverts et les mieux éclairés parmi le clergé et les leaders laïcs ont commencé à se rendre compte du besoin de respecter les traditions de l'autre groupe. Déjà en septembre 1958 j'ai pu écrire ... (Vol. 2, n. 1 p. 2) ...

«... Un principe directeur dans la création de ces comités a été le caractère bilingue de notre Diocèse. À notre avis, le vrai 
concept du bilinguisme est plus que le fait de pouvoir parler quelques mots dans une autre langue. C'est beaucoup plus fondamental. Le bilinguisme au Canada dans son vrai sens, veut dire compréhension et appréciation de l'histoire, des traditions et des aspirations d'un peuple. Ceux-là seulement qui font un effort réel pour saisir cette réalité peuvent se dire vraiment bilingues, si habiles soient-ils dans l'usage de la langue anglaise ou française. Nous pouvons dire que nous avons été grandement édifié par l'effort sincère du clergé de langue française et de langue anglaise de même que les fidèles, en vue de trouver un terrain commun d'entente afin de mieux travailler pour le bien des âmes, de l'Église et de la gloire de Dieu ...

« Si cet esprit continue de s'affermir et de se développer, il rendra un service immense à notre Église et à notre pays. Les plus éclairés de nos prêtres et de nos fidèles ont emboîté le pas; que les autres suivent leur exemple. Dans cette ligne de pensée, nous avons établi deux sections dans certains comités. Cela ne doit pas concourir à nous diviser. Au contraire, le plan d'ensemble reste le même; chaque section fonctionnera en harmonie avec l'autre, et au moins une fois par année les deux sections se rencontreront pour discuter de problèmes communs. Une telle réunion annuelle aura l'avantage de familiariser chaque groupe avec les problèmes de l'autre. Dans les différents mouvements d'Action Catholique et d'Action Sociale, soit par tradition ou à cause de la mentalité nationale, certaines formes d'action et certaines associations sont mieux adaptées aux besoins propres des Catholiques de langue anglaise ou de langue française respectivement. D'autres sont communes aux deux groupements et une plus grande coordination devrait exister entre eux. Dans tous ces mouvements cependant, doit régner un esprit de charité, car tous sont destinés à profiter aux âmes, et les fruits de ces activités devront enrichir tous les membres du Corps Mystique du Christ ».

3 - Pour remédier au chaos de la situation financière, il a fallu lancer une campagne de souscription. On sentait le besoin de prélever les fonds nécessaires pour solder les nouvelles entreprises, pour venir en aide à nos écoles et à nos institutions, pour payer des salaires convenables et pour créer un fonds de pension pour nos prêtres. La souscription devait en même temps faire l'éducation de nos gens. Les contributions étaient minimes si on prenait en considération le niveau des salaires. Cette campagne devait aider aussi aux paroisses qui étaient autorisées à retenir une bonne partie 
des fonds sollicités pour pourvoir à leurs propres besoins. Notre peuple a répondu avec une générosité superbe. L'expérience que nous avons vécue ensemble a consolidé le diocèse pendant ces premières années. Nous avons découvert parmi nos laïcs de nouveaux leaders.

$\mathrm{Au}$ début il y eut un peu d'hésitation de la part de la population francophone. Elle craignait toujours une déception blessante. De plus, certains individus de l'extérieur se mêlaient de l'affaire; vraisemblablement ils souffraient l'illusion qu'ils avaient une vocation divine pour diriger de loin leurs compatriotes. L'assurance que les fonds retourneraient à chaque secteur et qu'un compte rendu public serait publié et mis entre les mains de chaque diocésain dans les églises paroissiales mit fin à cette hésitation. Les maisons d'éducation, des institutions diocésaines, les paroisses, les œuvres dans chaque secteur ont bénéficié de cet effort commun dont les bienfaits durent encore. Au secteur français, le Collège Notre-Dame a reçu un montant considérable d'argent qui a permis aux Sœurs Grises de bâtir leur collège à Sudbury. North Bay, Verner, Noelville, Alban, Hanmer, Azilda ont profité de l'argent de la campagne de souscription surtout pour venir en aide à la cause de l'éducation française au stage secondaire. Le Collège du Sacré-Cœur a reçu une somme destinée à améliorer et à agrandir son édifice. Les argents perçus ont permis aussi la construction de la maison des Retraites Fermées à Long Lac et d'un gymnase pour les étudiantes du Couvent Notre-Dame de Lourdes à Sturgeon Falls. Si quelquesunes de ces institutions sont disparues aujourd'hui à ma grande tristesse, je vous l'avoue, je suis convaincu que toute cette expérience a créé une fierté chez nos francophones et leur a permis de prendre conscience du rôle qu'ils étaient appelés à jouer dans notre Église. La découverte d'hommes et de femmes de talent et de bonne volonté a donné naissance à de nouvelles initiatives. Dans les deux secteurs, je n'avais plus de difficulté à trouver des conseillers quand on a jugé le temps opportun de créer un comité diocésain de pastorale en 1960.

4 - Pendant ces premières années, deux événements ont aidé à nous convaincre du besoin de nous occuper de la dimension sociale. $\mathrm{La}$ grève à Sudbury fut un véritable défi. Nous avons mis sur pied une organisation diocésaine d'urgence pour s'occuper des familles éprouvées. L'intervention de l'Église nous a aidé à former une conscience sociale auprès de notre population. 
Le deuxième événement fut le Social Life Conference. La Conférence porta sur la famille. Pour la bien préparer, nous l'avions fait précéder d'une enquête sur la vie de famille. Nous avons eu recours à l'aide du renommé sociologue, le Père John L. Thomas, S.J. Depuis lors, les enquêtes sont monnaie courante. À cette époque, c'était du nouveau. Notre Conférence a mérité les éloges du Père Thomas; il a su nous en féliciter dans un de ses articles. Les mots exacts m'échappent mais il disait «at last a diocese has come of age socially $\gg$.

5 - Notre Église entière s'ouvrait donc de plus en plus et sentait le besoin de partager davantage. Nous étions en mesure de répondre à l'appel de Jean XXIII sollicitant tous les Catholiques de l'Amérique du Nord de venir en aide aux Églises de l'Amérique Latine. En 1961 nous avons accepté de pourvoir aux besoins de quelque 50,000 personnes dans le diocèse de Zacapa. La paroisse de Gualan et de La Union faisaient pitié. Grâce à la générosité de nos prêtres et de nos diocésains, nos prêtres et nos sœurs de St-Joseph qui ont pris part à cette initiative ont fait des merveilles. Nous en sommes fiers et je voudrais voir un jour une étude historique et même sociologique faite sur cette entreprise. Un tel document serait à la fois édifiant et révélateur.

\section{Notre Eglise pendant le Concile - 1962-1965}

$\mathrm{Au}$ moment de l'annonce du Concile, notre Église était déjà en marche. Nous venions aussi de fonder un journal diocésain, The Northern Register. Une version française allait suivre sous peu. Tout semblait serein et nous nous attendions à un développement mesuré, tranquille et soutenu. Voilà qu'on annonce un Concile ocuménique! Un coup d'éclair lancé par le Pape Jean XXIII, ce Pape qu'on avait caractérisé de pape transitoire! Au début, personne ne s'inquiétait. Moi-même, membre du comité pré-conciliaire de l'apostolat laï, je m'attendais à quelque chose de tranquille. Un Concile qui pourrait clarifier quelques questions théologiques controversées et qui donnerait quelques orientations ecclésiales mais qui laisserait l'Église jouir d'une paix et d'une tranquillité éminemment appréciée et du clergé et des fidèles.

Je n'ai pas évidemment à faire le bilan du Concile. Vous savez tous les bouleversements qu'il a causés dans l'Église et les suites imprévues auxquelles il a donné naissance. Je me contente de traiter de notre Église pendant ces années du Concile. 
a) Préparation au Concile :

Notre Église s'est intéressée au Concile sans trop savoir à quoi on devait s'attendre. Des questionnaires ont été préparés et envoyés dans les paroisses pour renseigner l'évêque sur les désirs, les espoirs et les aspirations des fidèles. Il devait se rendre à Rome non pas comme simple individu mais comme pasteur de son Église locale et il devait être le signe et l'instrument de l'union de cette Église avec toutes les Églises locales dans la sainte Église catholique sous la direction du Pontife Romain.

\section{b) Initiatives pendant le Concile :}

Les membres les mieux inspirés de notre Église se sont vite rendu compte que l'action de l'Esprit Saint au Concile ocuménique entraînerait des conséquences énormes pour notre Église locale. Même la première session qui fut plutôt une prise de position offrit tout de même un avenir riche en autorisant l'utilisation de la langue populaire pendant les célébrations de l'Eucharistie. On savait quand même qu'une telle décision serait lourde de conséquences chez nous pour les paroisses bilingues et les paroisses nationales.

À mesure que le Concile avançait, il devenait évident qu'ón assistait à un renouveau fondamental. Pour se mettre au diapason de ce renouveau de l'Église universelle, toute l'Église locale, non seulement l'évêque, devait participer de quelque façon à cette expérience de l'Église universelle. Nous avons donc décidé de mettre sur pied un Concile diocésain parallèle au Concile œcuménique. On le nomma le Petit Concile. Les commissions sont nommées, en tenant compte des distances qui séparent les grands centres et des différences de culture et de langue. Les discussions publiques s'organisent en conséquence. On est d'accord pour conduire des discussions parallèles aux discussions du Concile Vatican II tout en laissant une certaine latitude pour nous permettre d'aborder les problèmes locaux les plus urgents et quitte à adapter aux conditions locales la grande ligne de pensée du Concile Universel. Nous avions l'espoir de jouer ainsi notre rôle dans l'Église universelle et de donner suite dans notre Église à l'Esprit et à la vitalité qui ne manquerait assurément pas de surgir du Concile Vatican II. Le Pape en convoquant le Concile avait fait appel à toute l'Église de mieux faire voir au monde le vrai visage du Christ. Il semblait donc logique que nous aussi nous devrions essayer de présenter ce même vrai visage à tous ceux qui vivaient dans les confins de notre diocèse. 
C'est ainsi que nous avons suivi toutes les grandes positions prises par le Concile. Je ne peux pas prétendre que le Petit Concile a affecté tous nos diocésains. J'ai quand même l'impression qu'un bon nombre en a profité.

c) À part cette participation intéressante et prenante aux débats du Concile, deux autres événements ont marqué ces années du Concile. La nomination de Monseigneur Adolphe Proulx comme Évêque auxiliaire du Diocèse est venue comme un coup d'éclair pour les diocésains, sauf évidemment pour queques individus qui ont été consultés d'avance. Personne n'y songeait, personne ne s'y attendait. Pendant assez longtemps, j'avais pris en considération une telle démarche. La présence d'un évêque auxiliaire de langue et de culture française couronnerait et solidifierait la nouvelle confiance de la population française et leur donnerait davantage un sentiment de sécurité dont elle avait encore besoin. Sans rompre les liens qui m'uniraient au secteur français, la présence d'un tel auxiliaire me rendrait la tâche plus humaine et plus sereine. Ai-je besoin de vous dire l'affection que j'ai eue et que je garde pour sa personne? Sa présence et son œuvre ont enrichi le diocèse. Quelle consolation de voir le bien qu'il a pu accomplir ici, en Alexandria et le bien qu'il accomplit maintenant à Hull.

2 - Le deuxième événement fut la création de l'Université Laurentienne à Sudbury. L'Université catholique de Sudbury fut invitée à en constituer une partie très importante. De longues discussions avaient précédé cette démarche. Il y eut de l'opposition de la part des dirigeants de l'Association Catholique de l'Éducation de Langue Française d'Ontario. Les représentants de l'Université de Sudbury, les officiers de l'Association, les Évêques de Hearst et de Timmins se sont rencontrés à North Bay à l'invitation de l'Évêque du Sault Ste-Marie. Tous se sont exprimés librement, les pour et les contre ont été entendus. Tout en comprenant avec sympathie les craintes des délégués de l'Association, nous, sur qui tombait la responsabilité de l'éducation universitaire de nos jeunes du nord, avons cru nécessaire d'appuyer le projet du gouvernement de l'Ontario. J'exprime le désir qu'un jour votre Société fasse préparer un travail sur toute cette question. L'histoire de l'Université de Sudbury est un sujet fascinant. Mais passons.

\section{Notre Eglise après le Concile}

Les résultats du Concile se faisaient sentir bientôt après sa clôture. Chez-nous le Petit Concile avait préparé un peu les esprits. 
On se mit à l'œuvre en 1966. Deux initiatives s'avéraient urgentes. Il nous fallait un Conseil Presbytéral et un Synode Diocésain. Le premier engagerait davantage tout le presbytérium dans la voie indiquée par Vatican II. Le Synode nous permettrait une consultation globale du diocèse qui nous permettrait de discerner les meilleurs moyens de concrétiser les principes et l'esprit du Concile. C'est dans ce but que le Synode Diocésain fut convoqué pour 1968. Le Père Daniel Hannin, S.J., Docteur en Sociologie et missionnaire bien connu dans notre diocèse, accepta la charge de coordonnateur. En raison des particularités de notre diocèse, la plupart des discussions se faisaient au plan régional. Un comité central de coordination faisait le lien entre les régions. Dans chaque région un groupe de francophones et un groupe d'anglophones se réunissaient pour discuter et pour faire leur contribution. Les sessions générales aussi se conduisaient par secteur et les deux groupes se réunissaient vers la fin de l'après-midi.

Le Synode malgré ses déficiences - et il y en avait, - a certainement aidé à transformer notre Église. Vraiment, nous étions tous des amateurs. Trop de résolutions ont été acceptées. Le nombre seul sans parler des sommes d'argent qu'il aurait fallu trouver quelque part dépassait de loin nos possibilités. Néanmoins il en est sorti un projet d'organisation diocésaine qui permettrait à tous les membres de notre Église de se faire entendre. Je crois que ce Synode a justifié nos espérances malgré notre manque d'expertise en ce domaine. La liberté de parole fut assurée dès le début. Hommes, femmes, jeunes, tous pouvaient participer et ont participé. De plus nous avons invité les non-catholiques. Toutes les sessions plénières restaient ouvertes à qui voulait assister. C'était en effet un exercice de ce qu'on avait appelé au Concile L'ÉGLISE OUVERTE.

Plusieurs craignaient que le Synode pourrait diviser davantage notre population. Ces prophètes de malheurs avaient peur d'un débat public. D'autres craignaient que l'autorité diocésaine serait diminuée, que la foi des gens serait affaiblie, etc. Si quelques-uns ont été mal édifiés ou scandalisés de certaines opinions exprimées pendant les discussions je crois que, à la longue, le jugement et le bon sens de la grande partie des participants les ont finalement rassurés. Pour dire le moins, le Synode a réussi à alerter nos diocésains et à préparer les mentalités. On en est sorti mieux doué pour affronter les problèmes qui tôt ou tard se feraient sentir dans chaque paroisse, pour ne pas dire dans chaque famille. 
Le Sénat des Prêtres ou le Conseil Presbytéral lui aussi a joué un grand rôle en animant la vie de notre Église selon l'esprit et les enseignements de Vatican II. Les prêtres ont initié chez nous un cours de recyclage, sous forme de séminaires et d'ateliers de travail. lls ont aussi offert à ceux qui le désiraient la chance d'étudier en dehors du diocèse. Ceci leur a permis d'apprendre les grandes lignes de la théologie en termes contemporains et d'acquérir un nouvel aperçu du rôle du prêtre et de l'Église devant les problèmes complexes et difficiles de notre temps.

Malheureusement le manque de connaissance du français de la part de nos prêtres anglais, disons aussi le manque de compréhension de l'autre mentalité des deux côtés, se faisait sentir très vite au sein du Sénat des Prêtres. De plus l'organisation de chaque secteur était fort différente. La situation devenait un peu gênante. On a jugé mieux de se séparer, de former deux Conseils, quitte à s'unir au sommet pour discuter des problèmes communs. Des réunions générales de tous les prêtres aussi fourniraient l'occasion de discuter ensemble et de garder un lien d'unité et d'amitié parmi tous.

Pendant qu'on mettait sur pied cette structure, le diocèse recevait un coup inattendu. Monseigneur Proulx était nommé Évêque d'Alexandria en Ontario. Son départ laissait un vide profond et sérieux dans notre Église locale. La nomination de Monseigneur Despatie vint combler ce vide. Une fois de plus, nous avions un prêtre de chez-nous, le choix prépondérant du clergé local lors des consultations qui furent faites après la nomination de Monseigneur Proulx. Lui aussi a collaboré intimement au Synode et aux offices diocésains établis pour réaliser les buts du Concile. Jusqu'en 1973, comme Évêque Auxiliaire, il a accompli un travail de géant. Encore une fois Rome est intervenu et on le nomma évêque de Hearst. On se trouvait à jouer le rôle de Job et il fallait répéter : "Dieu donne et Dieu enlève - béni soit le nom de Dieu !» La Providence, cependant, veille sur nous. Une assemblée de tous les prêtres et le Conseil Pastoral Diocésain ont appuyé la suggestion d'avoir deux Auxiliaires à cause des particularités de notre diocèse et des grandes distances à parcourir entre les grands centres. Rome a accepté nos démarches après un certain retard à cause de la nature de notre demande et encore une fois elle nous a présenté deux hommes de grande envergure. Déjà la présence de Monseigneur Gérard Dionne et Monseigneur Bernard Pappin nous console et nous rassure. Nous sommes tous trois bien décidés de travailler ensemble dans un esprit 
d'amitié et de fraternité pour le bien de toute la population de notre milieu.

\section{Conclusion}

Où en sommes-nous dix ans après le Concile? Notre Eglise connaît des côtés sombres et des côtés lumineux.

\section{1 - Ombres et Anxiétés :}

Comme tous, nous avons souffert des bouleversements à la suite du Concile. Le départ de plusieurs prêtres a été une source de tristesse et de perturbation parmi une population si attachée à la personne et au rôle du prêtre. Nos congrégations religieuses d'hommes, de femmes aussi, ont subi des pertes sérieuses, pertes qui fatalement affectent les services qu'elles pouvaient rendre au peuple de Dieu.

2 -Notre population n'échappe pas aux influences toujours plus omniprésentes de notre siècle. Les valeurs chrétiennes sont ridiculisées; les extravagances de quelques théologiens nuisent à la foi en Jésus-Christ. Dans quelques endroits la pratique religieuse baisse. Le danger existe que la révolution sexuelle pourrait séduire nos jeunes voire notre peuple à des excès déplorables.

3 - Un danger encore plus subtil nous guette. Sous l'enseigne de Vatican II et sous préteexte de corriger les abus du passé, certains semblent avoir la tendance

a) de retomber dans un esprit étroit - un esprit de clocher. Une paroisse ou un groupe ésotérique se croit suffisant et oublie qu'il fait partie d'une Église locale et d'une Église universelle. La Una Catholica (St-Augustin) en souffre et l'on flnit par déformer la nature même de l'Église;

b) la tendance de négliger le côté sacrifice de l'Eucharistie et de manquer de révérence et de respect envers le Saint-Sacrement. On semble confondre fraternité et vulgarité !

c) la tendance d'ignorer complètement la place et le rôle de la Mère de Dieu dans la sainte Tradition et dans la foi de l'Église;

d) de rompre avec le passé et d'imposer ses propres idées (quelques fois un peu farfelues) sur le peuple de Dieu. 
En somme tous les dangers que n'importe quel Catholique avec un minimum de discernement peut découvrir dans l'Église de l'Ouest existent aussi chez-nous et il nous incombe d'en être avertis et d'y apporter notre attention.

\section{Lumières et promesses}

Par contre la transformation de notre Église dans le sens indiqué par Vatican II se poursuit et on en découvre des fruits immenses :

1 - Une foi beaucoup plus convaincue et profonde parmi un nombre croissant de nos fidèles. Ils sont fiers d'être catholiques et ils tiennent à remplir leur propre rôle dans l'Église.

2- Un culte qui commence enfin à célébrer notre foi. Il y a de la créativité; on exprime la foi en termes concrets, on partage ensemble, on s'aime davantage.

3 - La préparation à la réception des grands sacrements de l'Église - le Baptême, la Confirmation, l'Eucharistie, devient plus sérieuse et demande davantage la participation des parents et de la communauté. De même pour le sacrement de Pénitence-et le sacrement des malades. La communauté chrétienne devient plus consciente d'elle-même et de sa foi lors de ces grandes célébrations de réconciliation et de guérison. C'est beau, c'est émouvant, c'est prometteur.

4- La participation de nos laïcs à la vie de l'Église. Les ministres laïcs de l'Eucharistie dans les paroisses où ils ont reçu une catéchèse et une instruction convenable révèlent combien il leur plaît d'exercer leur sacerdoce commun au service de leurs frères. De plus en plus, nos laïcs s'engagent et nous venons d'initier des cours d'instruction religieuse en vue de former des ministres aptes à enrichir l'Église de leur esprit et de leur dévouement.

5 - La manifestation de la foi devient de plus en plus édifiante et prend des formes diverses. On prie ensemble - groupes charismatiques, cursillos, etc. On se rend compte davantage de son devoir de jouer un rôle social dans le monde et de chercher la justice pour tous; comme preuve je vous offre la réponse de nos diocésains aux déclarations du magistère au sujet du partage du pain, du tiersmonde, du développement du Nord : par exemple notre Carême Partage dépasse cent mille dollars; il a triplé dans l'espace de trois 
ans. C'est un signe. On peut y voir l'indice d'une conscience qui s'éveille davantage.

Voilà notre Église. Elle n'a pas de prétentions; elle se connaît telle qu'elle est - toujours exposée aux formes du mal et affectée par elles. Elle reconnait tout de même qu'il y a de beaux projets qui sont lancés, de belles initiatives qui se créent, des succès qui se réalisent. Étant du Nord de l'Ontario, les deux pieds fermement plantés sur le sol, elle a assez de bon sens pour savoir que tout cela ne vient pas seulement d'elle mais l'Esprit de Dieu qui vit et se meut en elle !

\author{
Alexander CARTER \\ Evêque de Sault-Sainte-Marie
}

